\title{
SENTIDO DE VIDA E SAÚDE MENTAL EM PROFESSORES: UMA REVISÃO INTEGRATIVA
}

\author{
Karine David Andrade Santos \\ Joilson Pereira da Silva \\ Universidade Federal de Sergipe, Aracaju-SE, Brasil
}

\section{RESUMO}

A presente revisão integrativa da literatura tem por objetivo investigar a relação entre sentido de vida (SV) e saúde mental em professores. As bases de dados acessadas para a busca das evidências foram: Web of Science - Coleção Principal, ERIC (ProQuest), PsycINFO, Scopus, SciELO e PePSIC. O período de publicação dos artigos abrangidos foi de janeiro de 2000 a dezembro de 2020 para responder à questão norteadora: Como a relação entre sentido de vida e a saúde mental em professores é abordada na literatura científica? Dos sete artigos recuperados, verificou-se que o sentido de vida está associado negativamente com síndrome de burnout e estresse percebido e proporciona melhores índices de funcionamento psicológico e de satisfação vital em docentes.

Palavras-chave: Logoterapia; Sentido de vida; Trabalho docente; Revisão de literatura; Professores.

\section{MEANING IN LIFE AND MENTAL HEALTH AT TEACHERS: AN INTEGRATIVE REVIEW}

\begin{abstract}
This integrative literature review aimed to investigate the relationship between the meaning of life and mental health in teachers. The database accessed for the search for evidence was: Web of Science - Main Collection, ERIC (ProQuest), PsycINFO, SciELO, and PePSIC. The articles covered were from January 2000 to December 2020 to answer the following guiding question: How is the relationship between the meaning of life and mental health in teachers addressed in the scientific literature? Of the seven selected articles, we found that the meaning of life is negatively associated with burnout syndrome and perceived stress and provides better rates of psychological functioning and vital satisfaction at teachers.
\end{abstract}

Keywords: Logotherapy; Meaning in life; Teaching work; Literature review; Teachers. 


\title{
SENTIDO DE VIDA Y SALUD MENTAL EN DOCENTES: UNA REVISIÓN INTEGRADORA
}

\begin{abstract}
RESUMEN
La presente revisión de literatura integradora tuvo como objetivo investigar la relación entre el sentido de la vida y la salud mental en los docentes. Las bases de datos a los que se accedió para buscar evidencia fueron: Web of Science - Main Collection, ERIC (ProQuest), PsycINFO, Scopus, SciELO y PePSIC. El período de publicación de los artículos fue de enero de 2000 a diciembre de 2020 para dar respuesta a la pregunta orientadora: ¿Cómo se aborda en la literatura científica la relación entre el sentido de la vida y la salud mental en los docentes? De los siete artículos seleccionados, se encontró que el sentido de la vida está asociado negativamente con el síndrome de quemarse y el estrés percibido y proporciona mejores índices de funcionamiento psicológico y satisfacción vital en profesores.
\end{abstract}

Palabras clave: Logoterapia; Sentido de vida; Trabajo docente; Metanálise; Profesores.

O trabalho docente é uma experiência profissional caracterizada por maiores níveis de estresse e baixos níveis de bem-estar, comparada a outras ocupações formais (Quiroga, 2015; Whitaker et al., 2015). No Brasil, a pesquisa Educatel realizada em 2017 para avaliar a saúde e as condições de trabalho da Educação Básica revelou que o estado de saúde e a capacidade para o trabalho dos professores estavam afetados de maneira significativa, principalmente pelas condições de trabalho (Alcantara et al., 2019), o que corroborou o índice de $71 \%$ de afastamento do trabalho por parte dos profissionais da educação pública, no mesmo período (CNTE, 2017). A Organização Internacional do Trabalho (OIT) considera a docência como atividade de risco desde 1981, fato que explica o baixo nível de atratividade pela profissão e crescente escassez dos profissionais de educação, principalmente, nos estágios iniciais de instrução escolar (Redding \& Henry, 2019).

A literatura tem indicado que circunstâncias internas e externas à sala de aula concorrem para o adoecimento psíquico e físico nos trabalhadores da educação, como: a) ergonômicas (pouca ventilação, altas temperaturas e ruídos internos e externos da sala de aula); b) financeiras (baixos salários e altas jornadas de trabalho; c) relacionais (conflitos com alunos e colegas de trabalho, baixo suporte social por parte dos gestores escolares e violência dentro da sala de aula); d) burocráticas (hierarquização e burocratização crescentes, pouca disponibilidade de tempo para o aprimoramento profissional e baixa autonomia profissional), dentre outras (Imbernón, 2011; Souza, 2017).

A pandemia da Covid-19 trouxe novos elementos estressantes para o trabalho docente, como o ensino remoto e a urgente necessidade de familiaridade com as ferramentas tecnológicas (Zaidan \& Galvão, 2020), aspectos que indicam como esse momento de emergência em saúde pública é um novo marcador macrossocial nos estudos da área. No entanto, a interpretação destes agentes estressores varia de pessoa para pessoa e de contexto para contexto (Aguiar \& Almeida, 2011), e sabe-se que essa variação no enfrentamento dessas condições se deve ao uso de recursos psicossociais, como o sentido de vida, um aspecto com efeito positivo na habilidade para lidar com eventos difíceis ou estressantes na vida (Heintzelman \& King, 2014). 
O sentido de vida ( $\mathrm{SV}$ ), como constructo psicológico, é um aspecto central apresentado e teorizado pela Logoterapia ou Análise Existencial (Frankl, 1969, 2011, 2015). Ao longo da obra frankliana, foi proposta uma nova dimensão do ser humano, a noética, distinta da psicológica e biológica, e responsável por instrumentar o psicofísico para a materialização da principal motivação humana: a busca do SV (Frankl, 2018).

O cumprimento do sentido de vida, pelo ser humano, está relacionado à autotranscendência, à autodeterminação, à liberdade e à responsabilidade, componentes da dimensão noética, que possibilitam a satisfação vital e a autorrealização (Frankl, 2015). Construções teóricas mais contemporâneas postulam que o sentido de vida é composto por três facetas: a) coerência (senso de que a vida faz sentido); b) propósito (ter objetivos e metas na vida); c) significado (grau em que a pessoa acredita que sua vida tem valor e importância) (Martela \& Steger, 2016; Park \& George, 2013).

Ao selecionar e classificar o que é importante na vida, o ser humano aprimora a coerência ou compreensão do mundo, alinhando os comportamentos com os objetivos e metas que preenchem o sentido de vida. Este destaque ou notabilidade do sentido da vida para a pessoa é denominado saliência de sentido, que influencia nas escolhas diárias, comportamentos, respostas às circunstâncias externas e, principalmente, na autorregulação, um aspecto crítico de saúde.

Ter maiores habilidades autorregulatórias reduz os efeitos estressores devido ao emprego de estratégias de enfrentamento adaptativas e de comportamentos de saúde (Hooker et al., 2018). Diferentes estudos indicam que encontrar sentido de vida está relacionado ao melhor estado e funcionamento geral da saúde física e mental (Czekierda et al., 2015; Damásio \& Koller, 2015; García-Alandete et al., 2018).

Considerando que a presença de SV é uma variável relacionada à promoção da saúde física e mental (Miao et al., 2017) e diante das evidências sobre as condições de estresse e os níveis de adoecimento em professores, tornou-se oportuno produzir um levantamento da literatura científica acerca das pesquisas sobre a relação entre sentido de vida ou realização existencial e saúde mental em professores. Estudos apontam que, em outras categorias profissionais, como médicos e bombeiros, a presença de sentido de vida tem um papel preventivo na manifestação de psicopatologias, como a síndrome de burnout (Hooker et al., 2020; Krok, 2016).

Para rastrear as informações existentes sobre sentido de vida em professores, foi elaborada uma revisão integrativa da literatura, considerada um recurso metodológico de coleta de informações importante na área da saúde, pois sintetiza dados teóricos e empíricos, que guiam a prática baseada em evidências (PBE) (Valdanha et al., 2013). Sendo assim, este estudo tem por objetivo investigar a relação entre sentido de vida (SV) e saúde mental em professores.

\section{MÉTODO}

Em alinhamento com os princípios da prática baseada em evidências - PBE, foi aplicada a estratégia PICO, para a construção da pergunta de pesquisa (Santos et al., 2007) que, segundo os autores, "PICO" representa um acrônimo para Paciente, Intervenção, Comparação e Resultado. Esses quatro componentes são os elementos essenciais da pergunta de pesquisa na PBE e da construção da pergunta para a busca bibliográfica de evidências. No presente estudo, ela é definida da seguinte forma: Como a relação entre sentido de vida e a saúde mental (I) em professores (P) é abordada na 
literatura científica $(\mathrm{O})$ ? Foi elaborada uma categorização dos estudos apresentados acerca de: a) aspectos bibliométricos, metodológicos e de conteúdo; b) relação entre sentido de vida e outras variáveis psicológicas; c) associação entre sentido de vida/realização existencial e condições de saúde; d) possíveis hiatos encontrados nos estudos selecionados, apresentando propostas para pesquisas.

O levantamento de dados para compor esta revisão integrativa foi realizado no período de maio de 2020, nas bases de dados Web of Science - Coleção Principal (Clarivate Analytics), Educational Resources Information Center - ERIC (ProQuest), Scopus (Elsevier), PsycINFO (APA), SciELO e PePSIC - Periódicos Eletrônicos em Psicologia, acessadas através do periódico CAPES, que dispõe da plataforma CAFE, uma via de acesso que alcança artigos em repositórios de dados conveniados. $\mathrm{O}$ rastreamento das produções científicas foi realizado, inicialmente, pelo uso das seguintes palavras-chave: "Meaning" or "Logotherapy" (todos os campos) and "Teachers" or "Teaching Work" (todos os campos), nas bases de dados selecionadas. Porém, os dados recuperados, nessa tentativa de busca, não respondiam ao questionamento central desta revisão. Na sequência, foi aplicada a seguinte exitosa combinação nos formulários avançados de pesquisa dos repositórios de dados selecionados: "logotherapy" or "meaning in life" or "existential fulfilment" or "purpose-in-life" and "Teachers" or "Teaching Work".

O descritor "Logotherapy" está indexado na Biblioteca Virtual em Saúde Psicologia (BVS-Psi); as demais palavras da primeira parte do cruzamento são palavraschave usuais da área pesquisada, utilizadas como parâmetros de busca, em outros estudos revisionais (Czekierda et al., 2015; Hupkens et al., 2018), e os termos da segunda parte da expressão foram extraídos dos Descritores em Ciência da Saúde (DeCS). As palavras da pesquisa foram manejadas no idioma inglês, nas bases internacionais, pelo método de busca "title", para a primeira parte da expressão de pesquisa, e da técnica de rastreamento "anyfield", para a segunda parte da combinação. Somente nos repositórios de dados Scielo e Pepsic, as expressões ("logotherapy" or "meaning in life" or "existential fulfilment" or "purpose-in-life") e ("purpose-in-life" and "Teachers" or "Teaching Work") e seus termos equivalentes em espanhol e português foram aplicados pelo método de busca "anyfield".

Os seguintes critérios de inclusão foram determinados para esta varredura: a) documento (apenas artigos empíricos); b) tempo (janeiro de 2000 a dezembro de 2020); c) idioma (manuscritos em inglês, espanhol e português); d) público (professores de qualquer nível de ensino); e) artigos empíricos elaborados em qualquer desenho metodológico; f) estudos realizados em seres humanos; g) acesso (disponíveis como texto completo pelo acesso aberto ou livre). Para os critérios de exclusão, os seguintes itens foram delimitados: a) documento (estudos que correspondessem à literatura cinzenta como teses, dissertações, livros, monografias, resenhas e produções similares); b) idioma (manuscritos redigidos em idiomas diferentes dos definidos nos critérios de inclusão); c) público (diverso do estabelecido no critério de inclusão); d) acesso restrito ou pago; e) estudos revisionais, teóricos ou similares; f) estudos realizados com animais; g) não estivesse disponível nas bases de dados selecionadas para esta investigação.

O rastreamento inicial obteve 616 registros, dos quais 377 eram oriundos da Web of Science - Coleção Principal (Clarivate Analytics) (61,2\%), 114 da Educational Resources Information Center - ERIC (ProQuest) (18,5\%), 62 da Scopus (Elsevier) $(10,1 \%), 57$ da PsycINFO (APA) $(9,2 \%), 3$ da SciELO (0,5\%) e 3 da PePSIC - 
Periódicos Eletrônicos em Psicologia (0,5\%). Para sistematização do processo seletivo dos manuscritos extraídos, conforme ilustrado na Figura 1, o método PRISMA foi aplicado como referência para esta atividade (Galvão et al., 2015). Durante a apreciação dos resumos e títulos dos artigos recuperados, foram eliminados: artigos duplicados $(n=$ $81)$, estudos que não tinham professores como público $(n=287)$, revisões $(n=12)$ e estudos com animais $(n=3)$.

Como resultado desta etapa de seleção, 233 manuscritos foram eleitos para a fase de avaliação de elegibilidade, etapa em que foram lidos na íntegra. 226 estudos foram excluídos nesta fase, pois não respondiam ao questionamento desta revisão. Após todas as etapas de seleção realizadas, com o apoio da plataforma Rayyan (Ouzzani et al., 2016) e da avaliação cega de um juiz, sete artigos foram eleitos para integrar a amostra deste estudo.

Para análise dos dados bibliométricos, metodológicos e de conteúdo, foi elaborada uma ficha específica. $\mathrm{Na}$ análise bibliométrica, foram consideradas as seguintes características dos manuscritos selecionados: ano, país de publicação, idioma e área de conhecimento. A análise metodológica sintetizou informações da amostra e dos instrumentos utilizados, a saber: delineamento do estudo, quantitativo da amostra, nível de ensino lecionado, idade e sexo dos participantes, e tipo de instrumento utilizado.

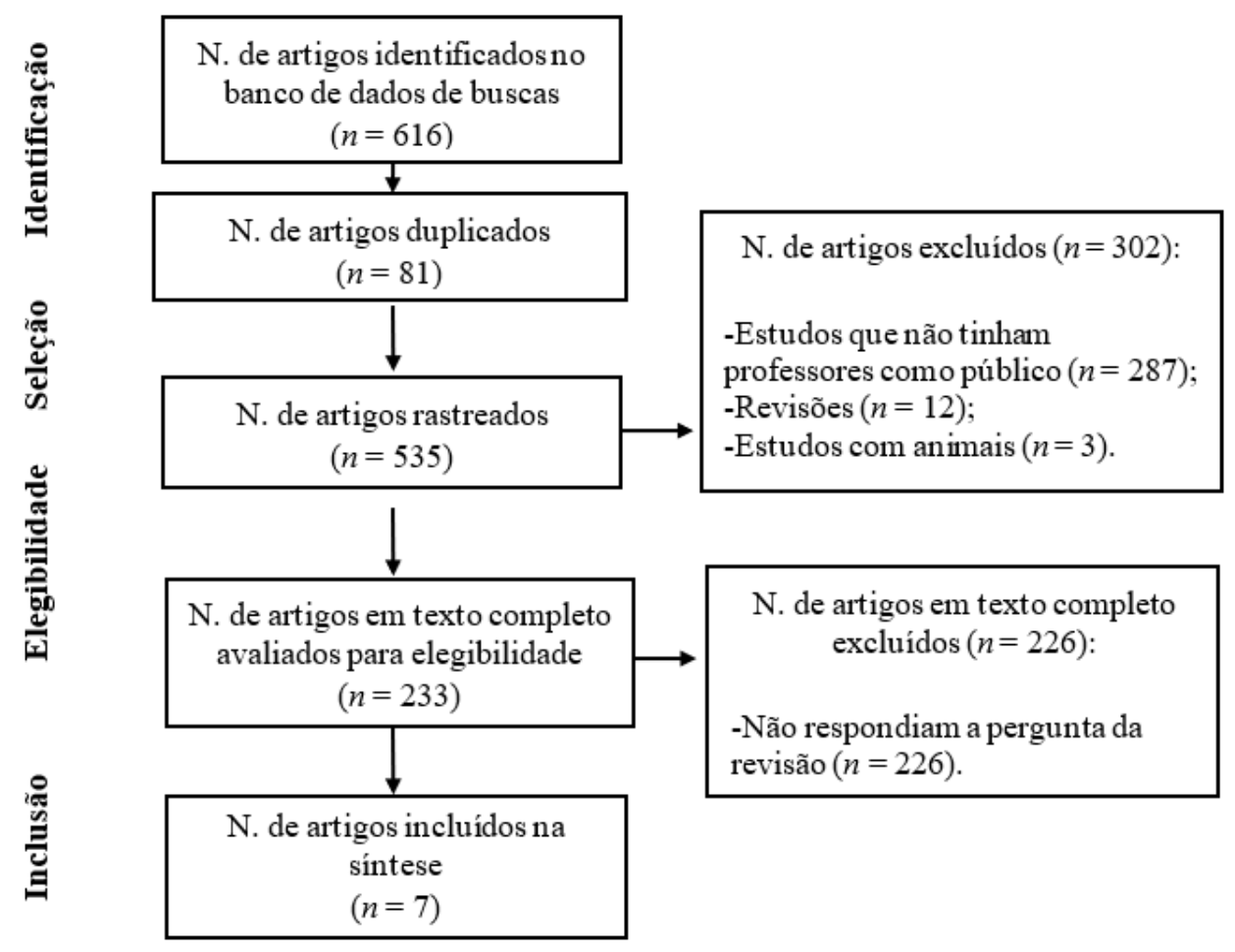

Figura 1

Fluxograma da seleção de artigos baseada no método Prisma 


\section{RESUltados}

\section{TÓPICOS BIBLIOMÉTRICOS E METODOLÓGICOS}

As publicações estavam concentradas nos intervalos de 2008-2013 e 2017-2018, a saber: um estudo em 2008 (E1: Tomic \& Tomic, 2008); dois em 2009 (E2: Loonstra et al., 2009; E3: Silva et al., 2009); um em 2013 (E4: Damásio et al., 2013); um em 2017 (E5: Liang et al., 2017) e dois em 2018 (E6: Li, 2018; E7: Taş \& İskender, 2018). Das pesquisas extraídas neste levantamento, três países concentravam as pesquisas de sentido de vida/realização existencial: Holanda (E1, E2), Brasil (E3 e E4) e China (E5 e E6). O idioma inglês foi utilizado para a redação das pesquisas E1, E2, E5, E6 e E7, e o português, para as investigações E3 e E4. Quatro artigos pertenciam a revistas científicas da área da Educação (E1, E2, E5 e E7) e três, da Psicologia (E3, E4 e E6).

Em relação ao delineamento dos estudos, seis artigos alojados nesta revisão tinham um delineamento transversal e um longitudinal. Ao totalizar a quantidade de pesquisados das sete publicações, 3.652 indivíduos foram investigados a fim de compreender a relação entre sentido de vida e outras variáveis psicológicas. A maior amostra foi localizada em um estudo com 610 professores, realizado na China, e a menor pertence a uma pesquisa realizada com 310 profissionais da educação. Um estudo foi realizado com professores e diretores (E1); um, com profissionais da educação do ensino médio (E2); um, com educadores do nível universitário (E6) e quatro, com professores do ensino fundamental e médio (E3, E4, E5 e E7).

Quanto à faixa etária, cinco estudos contemplaram participantes na faixa de 1665 anos, em média, e dois estudos não informaram este dado. No tocante ao sexo/gênero dos pesquisados, $100 \%$ dos estudos foram realizados com homens e mulheres, sendo predominante a participação feminina, em seis estudos (E1, E2, E3, E4, E5 e E6).

O sentido de vida foi avaliado em cinco estudos (E3, E4, E5, E6 e E7); associada à satisfação com a vida, em uma pesquisa (E7); com estresse percebido, em outra publicação (E3), e bem-estar psicológico, em três estudos (E4, E5, E6); enquanto, a realização existencial foi mensurada em duas pesquisas (E1 e E2). O Purpose in Life Test (PIL-Test-12) foi utilizado em dois estudos (E3 e E4); dois manuscritos (E6 e E7) aplicaram o Meaning in Life Questionnaire (MLQ); a Existence Scale (ES) foi instrumento de pesquisa em E1; a Existential Fulfilment Scale (EFS), em E2 e uma investigação (E5) coletou os dados de sentido de vida pelo uso do Teacher's Sense of Meaning in Life Scale. Já a realização existencial foi mensurada pelos instrumentos Existence Scale (ES) em E1, e Existential Fulfilment Scale (EFS), em E2.

\section{ANÁLISE DE CONTEÚDO}

\section{ASSOCIAÇÃO ENTRE SENTIDO DE VIDA E ESTRESSE}

A atual categoria de análise tem por finalidade condensar dados sobre a relação entre estresse percebido/ocupacional ou síndrome de burnout com a realização existencial/sentido de vida, em E1, E2 e E3. Nestas pesquisas, os desfechos foram mensurados por meio de métodos estatísticos inferenciais.

A pesquisa de 2009, no Brasil (E3), objetivou analisar como o estresse percebido se correlaciona com os índices de sentido de vida, e os resultados indicaram, por meio da correlação de Pearson, que o vazio existencial está correlacionado positivamente 
com o estresse percebido $(r=0,42)$ e a má administração de estresse $(r=0,47)$; enquanto que a presença de $\mathrm{SV}$, negativamente com o primeiro fator $(r=-0,42)$ e $\mathrm{o}$ segundo fator $(r=-0,51)$. Destaca-se que, nesta investigação, o sentido de vida foi apontado como agenciador do gerenciamento de estresse, explicando o menor índice deste fator.

Nos estudos realizados na Holanda, E1 e E2, buscou-se avaliar a relação entre os sintomas do estresse ocupacional e a realização existencial, utilizando a regressão hierárquica como método de análise. Na pesquisa de 2008, realizada com professores e diretores, foram apresentados os seguintes resultados similares para os grupos, respectivamente: a responsabilidade foi um preditor negativo da exaustão emocional ( $\beta$ $=-0,22 ; \beta=-0,38)$ e positivo da realização pessoal $(\beta=0,18)$, somente nos docentes; a autotranscedência prediz negativamente a despersonalização $(\beta=-0,23 ; \beta=-0,16)$; a liberdade está relacionada com o decréscimo da eficácia profissional $(\beta=-0,27 ; \beta=$ $0,17)$. Os resultados demonstraram uma correlação negativa entre a realização existencial e dois sintomas do estresse ocupacional, exaustão emocional, e positiva com a realização pessoal reduzida mesmo em grupos alocados em funções distintas no ambiente escolar.

$\mathrm{Na}$ investigação de 2009 (Loonstra et al., 2009), no mesmo país, realizada somente com professores do ensino médio com uma escala de três fatores existenciais: autoaceitação, autodistanciamento e autotranscendência, foi destacado que estes três aspectos são preditores positivos da realização profissional $(\beta=0,17 ; \beta=0,36 ; \beta=$ 0,11 ) ao passo que, autoaceitação e autorrealização constituem preditores negativos da exaustão emocional e da despersonalização. Os achados apresentaram o papel amortecedor destes componentes no burnout. Ao cruzar os resultados de E2 e E1, identifica-se que não há uma intersecção dos achados relativos de cada fator e os sintomas da síndrome de burnout. No entanto, os três estudos analisados convergem para a correlação negativa entre a realização existencial e a presença de sintomas do estresse ocupacional ou de estresse percebido, ou seja, índices elevados dos componentes da dimensão noética, responsável pelo sentido de vida, minimiza a manifestação do estresse.

\section{ASSOCIAÇÃO ENTRE SENTIDO DE VIDA E BEM-ESTAR PSICOLÓGICO}

Esta categoria sumarizou as informações relativas as pesquisas E4, E5, E6 e E7 que associam sentido de vida e bem-estar psicológico (BEP). As pesquisas desta seção também buscaram revelar a relação da SV com outros constructos, como qualidade de vida, capital psicológico, espiritualidade no local de trabalho, satisfação com a vida e autoconceito.

Nos estudos E4, E5 e E7, o bem-estar psicológico e o sentido de vida estão relacionados positivamente, através de modelos estatísticos inferenciais. A pesquisa no Brasil em 2013, que avaliou os índices de sentido de vida (SV), de bem-estar psicológico (BEP) e de qualidade de vida (QV) em uma ampla amostra de professores escolares, e observou como o SV poderia atuar como variável moderadora da relação entre o BEP e a QV geral, identificou, através da regressão linear, as predições positivas do $\mathrm{BP}(\beta=0,33)$ e do SV $(\beta=0,21)$ na qualidade de vida dos pesquisados. Em professores com baixo sentido de vida, há uma relação significativa entre bem-estar psicológico e qualidade de vida, indicando que o SV constituía um moderador da relação entre BP e qualidade de vida. 
$\mathrm{Na}$ investigação de 2017, realizada na China, com a finalidade de analisar a relação da espiritualidade, no local de trabalho dos professores, o sentido do significado na vida e o bem-estar psicológico, os resultados obtidos pela regressão hierárquica apontaram que as três dimensões para uma vida significativa, liberdade de vontade ( $\beta$ $=0,16)$, vontade de sentido, $(\beta=0,15)$ e sentido de vida $(\beta=0,27)$, estavam associadas à espiritualidade no local de trabalho $(\beta=0,05)$, e, conjuntamente, tinham um forte poder preditivo $(\beta=0,62)$ para o bem-estar psicológico. Assim como apontado em E4, esta pesquisa aponta que ter um senso de vida significativa ou ter sentido de vida contribui para bons índices de bem-estar psicológico.

Já outro estudo longitudinal, no mesmo país em 2018, objetivando abordar a precedência temporal entre capital psicológico (Psycap) e sentido de vida (SV), e examinar a relação entre sentido de vida, capital psicológico e bem-estar (BP) entre professores universitários, apontou, pela regressão hierárquica e modelo de equações estruturais, que o SV mediou parcialmente o efeito do capital psicológico no BP entre professores universitários, ao longo do tempo, sendo que o capital psicológico é um preditor do sentido de vida $\left(\chi 2(\mathrm{df}=1946)=8412,42 ; \chi^{2} / \mathrm{df}=4,32 ; \mathrm{RMSEA}=0,055\right.$; SRMR $=0,057$; AGFI $=0,916$; NNFI $=0,932 ; \mathrm{CFI}=0,935)$, mas não o inverso. Sendo assim, há uma divergência parcial desta pesquisa com as demais no tocante ao poder inferencial do sentido de vida em relação ao bem-estar psicológico.

Em investigação na Turquia, em 2018, realizada para examinar o sentido de vida, satisfação com a vida, autoconceito e lócus de controle entre professores, surgiram como principais resultados, por meio da correlação de Pearson, que ter sentido de vida se correlaciona positivamente com a satisfação com a vida $(r=0,141)$, autoconceito $(r$ $=0,333)$, e negativamente com lócus de controle $(r=-0,171)$. Nesse estudo, foi observado que os índices de busca de sentido diferiam significativamente por gênero e por estado civil, sendo maiores em mulheres e profissionais casados; ao passo que este efeito não foi encontrado em relação aos de presença de sentido. Além de trazer variáveis psicológicas distintas de E4, E5 e E6, esse foi o único que, nessa subseção, considerou variáveis sociodemográficas, como o gênero e o estado civil dos participantes, em suas análises de dados.

Em suma, as pesquisas apontam para os bons índices de bem-estar psicológico, qualidade de vida, satisfação com a vida e autoconceito promovidos pela presença do sentido de vida nos indivíduos. Esta variável contribui para uma experiência positiva do viver, a manutenção e preservação da saúde e a minimização de sintomas de estresse e de outras psicopatologias advindas do contato com eventos estressantes. As principais informações dos estudos ora apresentados estão contidas na Tabela 1.

\section{Tabela 1}

Principais informações das características bibliométricas, metodológicas e de conteúdo dos estudos recuperados na revisão.

\begin{tabular}{|c|c|c|c|}
\hline Estudo (I) & Amostra (P) & Objetivos (I) & Resultados (O) \\
\hline $\begin{array}{c}\text { E1: } \\
\text { Tomic e } \\
\text { Tomic } \\
(2008) .\end{array}$ & $\begin{array}{l}215 \text { professores e } \\
514 \text { diretores } \\
\text { (Holanda, } \\
\text { Professores - } \\
79,5 \% \text { e } \\
\text { Diretores- } 24 \% \\
\text { ). }\end{array}$ & $\begin{array}{l}\text { Examinar a prevalência de } \\
\text { burnout e a realização } \\
\text { existencial entre professores da } \\
\text { escola fundamental e diretores } \\
\text { de escolas que trabalham no } \\
\text { ensino primário e avaliar a } \\
\text { relação entre realização } \\
\text { existencial e dimensões de } \\
\text { burnout. }\end{array}$ & $\begin{array}{l}\frac{\text { Regressão hierárquica }}{\text { Responsabilidade - preditor negativo }} \\
\text { da exaustão emocional }(\beta=-0,22) \text {. } \\
\text { Autotranscendência - preditor } \\
\underline{\text { negativo da despersonalização }(\beta=} \\
-0,23) \text {. } \\
\text { Liberdade - preditor negativo da } \\
\text { realização profissional reduzida }(\beta= \\
-0,27) \text {. }\end{array}$ \\
\hline
\end{tabular}




\begin{tabular}{|c|c|c|c|}
\hline Estudo (I) & Amostra (P) & Objetivos (I) & Resultados $(\mathrm{O})$ \\
\hline $\begin{array}{l}\text { E2: } \\
\text { Loonstra et } \\
\text { al. }(2009) \text {. }\end{array}$ & $\begin{array}{l}504 \text { professores do } \\
\text { ensino médio } \\
\text { (Holanda, } 54,7 \% \\
\text { o). }\end{array}$ & $\begin{array}{lcr}\text { Avaliar a } & \text { relação entre } \\
\text { realização } & \text { existencial } & \text { e } \\
\text { esgotamento } & \text { entre professores } \\
\text { do ensino } & \text { secundário na } \\
\text { Holanda. } & & \end{array}$ & $\begin{array}{l}\text { Regressão hierárquica } \\
\text { Autoaceitação, autodistanciamento e } \\
\text { autotranscendência }- \text { preditores } \\
\text { positivos da realização profissional } \\
(\beta=0,17 ; \beta=0,36 ; \beta=0,11) \text {. } \\
\text { Autoaceitação e autorrealização - } \\
\text { preditores negativos da exaustão }(\beta= \\
0,50 ; \beta=0,18) \text { e a despersonalização } \\
(\beta=0,40 ; \beta=0,09) \text {. }\end{array}$ \\
\hline $\begin{array}{l}\text { E3: } \\
\text { Silva et al. } \\
\text { (2009). }\end{array}$ & $\begin{array}{l}517 \text { professores do } \\
\text { ensino } \\
\text { Fundamental e } \\
\text { médio (Brasil, } \\
65,89 \% \text { o). }\end{array}$ & $\begin{array}{l}\text { Realizar um levantamento } \\
\text { acerca dos índices de estresse } \\
\text { percebido entre professores do } \\
\text { Ensino Fundamental e do } \\
\text { Ensino Médio da cidade de } \\
\text { Campina Grande, PB, além de } \\
\text { analisar como o estresse } \\
\text { percebido correlaciona-se com } \\
\text { os índices de sentido de vida. }\end{array}$ & $\begin{array}{l}\text { Correlação de Pearson } \\
\text { A realização existencial se } \\
\text { correlacionou negativamente com o } \\
\text { estresse percebido }(r=-0,42) \text {. }\end{array}$ \\
\hline $\begin{array}{l}\text { E4: } \\
\text { Damásio et } \\
\text { al. (2013). }\end{array}$ & $\begin{array}{l}517 \text { professores, } \\
\text { de } 57 \text { escolas } \\
\text { públicas e privadas } \\
\text { (Brasil, } 343 \text { ㅇ). }\end{array}$ & $\begin{array}{l}\text { Avaliar os índices de sentido de } \\
\text { vida (SV), de bem-estar } \\
\text { psicológico (BEP) e de } \\
\text { qualidade de vida (QV) em } \\
\text { uma ampla amostra de } \\
\text { professores escolares, e } \\
\text { observar como o SV poderia } \\
\text { atuar como variável } \\
\text { moderadora da relação entre o } \\
\text { BEP e a geral. }\end{array}$ & $\begin{array}{l}\text { Regressão Linear Simples } \\
\text { Relação significativa entre o BEP e } \\
\text { QV geral para os professores com } \\
\text { baixo SV }(\beta=0,5, \mathrm{t}(95)=5,753) \text {. O } \\
\text { SV moderou a relação entre BEP e } \\
\text { QV. }\end{array}$ \\
\hline $\begin{array}{l}\text { E5: } \\
\text { Liang et al. } \\
\text { (2017). }\end{array}$ & $\begin{array}{l}610 \text { professores de } \\
\text { diferentes níveis } \\
\text { de ensino escolar } \\
\text { (China, } 65,9 \% \text { \%). }\end{array}$ & $\begin{array}{l}\text { Analisar a relação entre a } \\
\text { espiritualidade no local de } \\
\text { trabalho dos professores, o } \\
\text { sentido do significado na vida e } \\
\text { o bem-estar psicológico. }\end{array}$ & $\begin{array}{l}\text { Regressão hierárquica } \\
62,70 \% \text { de poder explicativo dos } \\
\text { preditores (espiritualidade no local } \\
\text { de trabalho e sentido de vida) para a } \\
\text { variável para o bem-estar } \\
\text { psicológico, sendo liberdade de } \\
\text { vontade }(\beta=0,16) \text {, vontade de } \\
\text { sentido }(\beta=0,15) \text { e sentido de vida } \\
(\beta=0,27) \text {. }\end{array}$ \\
\hline $\begin{array}{c}\text { E6: } \\
\text { Li (2018). }\end{array}$ & $\begin{array}{l}412 \text { professores } \\
\text { universitários } \\
\text { (China, 27,75\% } \\
\text { क). }\end{array}$ & $\begin{array}{l}\text { Abordar a questão da } \\
\text { precedência temporal que } \\
\text { vincula capital psicológico } \\
\text { (Psycap) e sentido de vida (SV) } \\
\text { e, além disso, examinar a } \\
\text { relação entre sentido de vida, } \\
\text { capital psicológico e bem-estar } \\
\text { (BP) entre professores } \\
\text { universitários. }\end{array}$ & $\begin{array}{l}\text { Modelo de Equação Estrutural } \\
\text { Psycap é um preditor positivo de SV } \\
\text { ao longo do tempo }(\chi 2(\mathrm{df}=1946)= \\
8412,42 ; \chi 2 / \mathrm{df}=4,32 ; \mathrm{RMSEA}= \\
0,055 ; \mathrm{SRMR}=0,057 ; \mathrm{AGFI}= \\
0,916 ; \mathrm{NNFI}=0,932 ; \mathrm{CFI}=0,935) \text {, } \\
\text { mas não o contrário. O SV mediou } \\
\text { parcialmente o efeito do Psycap no } \\
\text { BP entre professores universitários, } \\
\text { ao longo do tempo. }\end{array}$ \\
\hline $\begin{array}{l}\text { E7: } \\
\text { Taş e } \\
\text { İskender } \\
(2018) \text {. }\end{array}$ & $\begin{array}{l}363 \text { professores de } \\
\text { diferentes níveis } \\
\text { de ensino escolar } \\
\text { (Turquia, } 40 \%, \\
\text { sem informação } \\
\text { sobre } \\
\begin{array}{l}\text { distribuição de } \\
\text { professores por } \\
\text { gênero). }\end{array}\end{array}$ & $\begin{array}{l}\text { Examinar o sentido de vida, } \\
\text { satisfação com a vida, } \\
\text { autoconceito e lócus de } \\
\text { controle entre professores por } \\
\text { várias variáveis. }\end{array}$ & $\begin{array}{l}\text { Correlação de Pearson } \\
\text { Ter sentido de vida se correlaciona } \\
\text { positivamente com a satisfação com } \\
\text { a vida }(r=0,141) \text { e autoconceito }(r \\
=0,333) \text { e negativamente com lócus } \\
\text { de controle }(r=-0,171) \text {. }\end{array}$ \\
\hline
\end{tabular}

Nota. Todos os resultados apresentados foram estatisticamente significativos $(\mathrm{p}<0,05)$. 


\section{DISCUSSÃO}

Os estudos recuperados na presente exploração foram produzidos nos últimos cinco anos, sendo que boa parte das publicações está concentrada no intervalo de 2017 2018, atestando o gradual incremento desta área de pesquisa e a pertinência de estudos que avaliem o sentido de vida em professores. Verifica-se a necessidade de retomar esta temática de investigação no Brasil, tendo em vista que o sentido de vida exerce um papel de promoção do bem-estar em trabalhadores, principalmente, de minimizar o sofrimento mental.

Outra questão a ser destacada é concernente a futuras ampliações da publicidade destas pesquisas por parte dos periódicos de Psicologia, pois as revistas da área de Educação prevaleceram neste levantamento. Quanto ao delineamento dos estudos, observou-se que somente um estudo foi longitudinal (E6), o que indica a demanda de mais pesquisas que avaliem como o sentido de vida impacta outras variáveis ou constructos psicológicos ao longo do tempo (Czekierda et al., 2019; Tostes et al., 2018).

A amostra de pesquisa dos estudos era, majoritariamente, composta por professores do nível fundamental e médio; apenas um dos estudos (E6), na China, teve os professores de nível universitário como amostra da investigação, revelando a necessidade de produções científicas, no Brasil, que pesquisem a relação entre saúde e sentido de vida em educadores universitários. Sabe-se que a precarização das condições laborais (ex: perdas de garantias trabalhistas e previdenciárias), o aumento da carga de trabalho e a infraestrutura insuficiente acarretam repercussões negativas para a saúde dos professores brasileiros, deste nível de ensino (Baptista et al., 2019; Leite \& Nogueira, 2017). Como o sentido de vida apresenta o potencial de reduzir o estresse, os impactos de eventos negativos (Park \& Baumeister, 2017) e de promoção da saúde (Hooker et al., 2018), esta é uma variável relevante a ser investigada, associada a indicadores de saúde, de estratégias de enfrentamento e de condições de trabalho, em profissionais da educação das universidades brasileiras.

Os estudos concernentes à associação entre estresse e sentido de vida (E1, E2 e E3) apontaram que, ter uma vida significativa ou ser realizado existencialmente, está relacionado a menores índices de estresse percebido e de manifestação dos sintomas da síndrome de burnout, assim como evidenciados em outros estudos (Hooker et al., 2020; Pulopulos \& Kozusznik, 2018). Sobre o estresse ocupacional ou síndrome de burnout, a logoterapia a entende como uma manifestação do vazio existencial, visto que os sintomas manifestados exteriorizam o envolvimento do profissional em atividades, nas quais não sentem ou perderam o sentido, levando à exaustão, à despersonalização e à ineficácia profissional (Längle, 2003). Ao passo que, aqueles que reportam uma vida realizada existencialmente, ou com sentido, conseguem interpretar e organizar os eventos estressantes de forma mais saudável, por meio de estratégias de coping e de habilidades autorregulatórias, o que proporciona uma perspectiva predizível e controlável da circunstância, contribuindo para a minimização dos níveis de estresse (Heintzelman \& King, 2014; Park \& Baumeister, 2017).

Sobre a associação entre SV e bem-estar psicológico (E4, E5, E6 e E7), as pesquisas dessa investigação consolidaram os achados de outras evidências científicas (Damásio \& Koller, 2015; Ho et al., 2010). A presença de sentido de vida foi um moderador na relação entre BEP e QV (E4), um preditor positivo para o bem-estar psicológico associado à espiritualidade no local de trabalho (E5), um mediador parcial 
do capital psicológico no BEP (E6) e correlacionado com a satisfação vital e autoconceito (E7).

Por diferentes vias, o SV contribuiu para o bem-estar psicológico dos participantes, uma avaliação pessoal do indivíduo sobre suas experiências estressantes ou negativas e habilidade para manejá-las. As pessoas realizadas existencialmente acreditam ser livres e responsáveis por ter uma vida preenchida de propósito e metas significativas para sua existência, o que desdobra um funcionamento humano emocional, cognitivo e motivacional positivo (Frankl, 1969, 2011; George \& Park, 2016). Uma vida com significado e objetivos traz um senso de controle e predição sobre os eventos vitais, possibilitando uma melhor ponderação das circunstâncias negativas ou estressantes por parte do indivíduo, isto é, um maior bem-estar psicológico (Burrow et al., 2014; Mascaro \& Rosen, 2006).

\section{CONSIDERAÇÕES FINAIS}

Em suma, destacou-se a necessidade de retomada de pesquisas com a temática investigada, no Brasil: a concentração de estudos em professores, em níveis de ensino fundamental e médio. Verificou-se que o sentido de vida ou realização existencial esteve relacionado negativamente com desfechos negativos de saúde, em professores, como estresse percebido e síndrome de burnout, que podem ocasionar prejuízos à vida e à carreira profissional destes trabalhadores e impactos negativos no ambiente escolar.

Por outro lado, uma vida significativa reflete melhores índices de funcionamento psicológico e de satisfação vital que, direta ou indiretamente, estavam relacionados a melhores índices de bem-estar psicológico, o que atesta a importância do SV como mecanismo de promoção da saúde mental. $\mathrm{O}$ exame das pesquisas recuperadas, neste levantamento, possibilitou a aproximação dos manuscritos elaborados sobre o tema, agregando achados que serão provas baseadas em evidências (PBE), para a prática profissionais da psicologia e demais trabalhadores da área da educação e saúde.

Acerca das limitações da presente revisão, o uso de artigos disponibilizados nas bases indexadas pelo periódico CAPES pressupõe que este levantamento não representa o universo completo de artigos sobre a temática pesquisada. Como segunda limitação, as diferentes estatísticas inferenciais e variáveis mensuradas, associadas ao bem-estar psicológico e sentido de vida, impossibilitaram um cruzamento comparativo dos indicadores trazidos pelos estudos (E3, E4, E5 e E7).

Por fim, considera-se relevante a elaboração de pesquisas que avaliem, conjuntamente, os transtornos psíquicos (ex: síndrome de burnout), a saliência de sentido e o sentido de vida em professores, e proponham um modelo teórico de compreensão dos fenômenos investigados dentro de uma perspectiva logoterapêutica. Destaca-se que a retomada dos estudos, nesta área, no país, pode ser realizada com o objetivo de testar modelos teóricos, como o sugerido acima, por meio de propostas interventivas voltadas para a saúde mental deste público, cumprindo um papel social e de novas perspectivas teóricas e práticas sobre a saúde mental dos professores. 


\section{REFERÊNCIAS}

Aguiar, M. R. A., \& Almeida, S. F. C. (2011). Mal-estar na educação: O sofrimento psíquico dos professores. Juruá.

Alcantara, M. A. D., Medeiros, A. M. D., Claro, R. M., \& Vieira, M. D. T. (2019). Determinantes de capacidade para o trabalho no cenário da Educação Básica do Brasil: Estudo Educatel, 2016. Cadernos de Saúde Pública, 35, 1-13. https://doi.org/10.1590/0102-311X00179617

Baptista, M. N., Soares, T. F. P., Raad, A. J., \& Santos, L. M. (2019). Burnout, estresse, depressão e suporte laboral em professores universitários. Revista Psicologia $\begin{array}{llll}\text { Organizações } & e & \text { Trabalho, } & \text { 19(1), }\end{array}$ http://dx.doi.org/10.17652/rpot/2019.1.15417

Burrow, A. L., Sumner, R., \& Ong, A. D. (2014). Perceived change in life satisfaction and daily negative affect: The moderating role of purpose in life. Journal of Happiness Studies, 15(3), 579-592. https://doi.org/10.1007/s10902-013-9436-9

CNTE. (2017). Dossiê: Privatização da e na educação: Projetos societários em disputa. Retratos da Escola/Escola de Formação da Confederação Nacional dos Trabalhadores em Educação (Esforce). Brasília: Autor. http://dx.doi.org/10.22420/rde.v11i21.831

Czekierda, K., Banik, A., Park, C. L., \& Luszczynska, A. (2015). Meaning in life and physical health: Systematic review and meta-analysis. Health Psychology Review, 11(4), 387-418. https://doi.org/10.1080/17437199.2017.1327325

Czekierda, K., Horodyska, K., Banik, A., Wilhelm, L., Knoll, N., \& Luszczynska, A. (2019). Meaning in life and physical quality of life: Cross-lagged associations during inpatient rehabilitation. Rehabilitation Psychology, 64(4), 425-434. https://doi.org/10.1037/rep0000281

Damásio, B. F., \& Koller, S. H. (2015). How search for meaning interacts with complex categories of meaning in life and subjective well-being?. The Spanish Journal of Psychology, 18(4), 1-10. https://doi.org/10.1017/sjp.2015.1

Damásio, B. F., Melo, R. L. P. D., \& Silva, J. P. D. (2013). Sentido de vida, bem-estar psicológico e qualidade de vida em professores escolares. Paidéia (Ribeirão Preto), 23(54), 73-82. https://doi.org/10.1590/1982-43272354201309

Frankl, V. E. (1969, 2011). A vontade de sentido: Fundamentos e aplicações da logoterapia. (I. S. Pereira, Trad.). Paulus.

Frankl, V. E. (2015). O sofrimento de uma vida sem sentido: Caminhos para encontrar a razão de viver (1a. ed.). É Realizações.

Frankl, V. E. (2018). A psicoterapia para todos. Vozes.

Galvão, T. F., Pansani, T. D. S. A., \& Harrad, D. (2015). Principais itens para relatar Revisões sistemáticas e Meta-análises: A recomendação PRISMA. Epidemiologia e Serviços de Saúde, 24(2), 335-342. http://dx.doi.org/10.5123/S167949742015000200017

García-Alandete, J., Martínez, E. R., Sellés Nohales, P., \& Soucase Lozano, B. (2018). Meaning in life and psychological weil-being in spanish emerging adults. Acta colombiana de Psicología, 21(1), 196-216. https://www.redalyc.org/jatsRepo/798/79855072009/79855072009.pdf

George, L. S., \& Park, C. L. (2016). Meaning in life as comprehension, purpose, and mattering: Toward integration and new research questions. Review of General Psychology, 20(3), 205-220. https://doi.org/10.1037/gpr0000077 
Heintzelman, S. J., \& King, L. A. (2014). Life is pretty meaningful. American Psychologist, 69, 561-574. https://doi.org/10.1037/a0035049

Ho, M. Y., Cheung, F. M., \& Cheung, S. F. (2010). The role of meaning in life and optimism in promoting well-being. Personality and Individual Differences, 48(5), 658-663. https://doi.org/10.1016/j.paid.2010.01.008

Hooker, S. A., Masters, K. S., \& Park, C. L. (2018). A meaningful life is a healthy life: A conceptual model linking meaning and meaning salience to health. Review of General Psychology, 22(1), 11-24. https://doi.org/10.1037/gpr0000115

Hooker, S., Post, R., \& Sherman, M. (2020). Awareness of meaning in life is protective against burnout among family physicians: A CERA study. Family Medicine, 52(1), 11-16. https://pubmed.ncbi.nlm.nih.gov/31689355/

Hupkens, S., Machielse, A., Goumans, M., \& Derkx, P. (2018). Meaning in life of older persons: An integrative literature review. Nursing Ethics, 25(8), 973-991. https://doi.org/10.1177/0969733016680122

Imbernón, F. (2011). Formação docente e profissional: Formar-se para a mudança e a incerteza. Cortez.

Krok, D. (2016). Can meaning buffer work pressure? An exploratory study on styles of meaning in life and burnout in firefighters. Archives of Psychiatry and Psychotherapy, 1, 31-42. http://dx.doi.org/10.12740/APP/62154

Längle, A. (2003). Burnout-Existential meaning and possibilities of prevention. European Psychotherapy, 4(1), 107-121. https://www.existenzanalyse.org/wpcontent/uploads/Burnout-Europ-Psychoth-2003.pdf

Leite, A. F., \& Nogueira, J. A. D. (2017). Fatores condicionantes de saúde relacionados ao trabalho de professores universitários da área da saúde: Uma revisão integrativa. Revista Brasileira de Saúde Ocupacional, 42, 1-15. https://doi.org/10.1590/23176369000010116

Li, Y. (2018). Building well-being among university teachers: The roles of psychological capital and meaning in life. European Journal of Work and Organizational Psychology, 27(5), 594-602. https://doi.org/10.1080/1359432X.2018.1496909

Liang, J. L., Peng, L. X., Zhao, S. J., \& Wu, H. T. (2017). Relationship among workplace spirituality, meaning in life, and psychological well-being of teachers. Universal Journal of Educational Research, 5(6), 1008-1013. http://www.hrpub.org/journals/jour_archive.php?id=95\&iid=1173

Loonstra, B., Brouwers, A., \& Tomic, W. (2009). Feelings of existential fulfilment and burnout among secondary school teachers. Teaching and Teacher Education, 25(5), 752-757. https://doi.org/10.1016/j.tate.2009.01.002

Martela, F., \& Steger, M. F. (2016). The three meanings of meaningin life: Distinguishing coherence, purpose, and significance. The Journal of Positive Psychology, 11, 531-545. https://doi.org/10.1080/17439760.2015.1137623

Mascaro, N., \& Rosen, D. H. (2006). The role of existential meaning as a buffer against stress. Journal of Humanistic Psychology, 46(2), 168-190. https://doi.org/10.1177\%2F0022167805283779

Miao, M., Zheng, L., \& Gan, Y. (2017). Meaning in life promotes proactive coping via positive affect: A daily diary study. Journal of Happiness Studies, 18(6), 16831696. https://doi.org/10.1007/s10902-016-9791-4 
Ouzzani, M., Hammady, H., Fedorowicz, Z., \& Elmagarmid, A. (2016). Rayyan - A web and mobile app for systematic reviews. Systematic Reviews, 5, 1-10. https://doi.org/10.1186/s13643-016-0384-4

Park, J., \& Baumeister, R. F. (2017). Meaning in life and adjustment to daily stressors. The Journal of Positive Psychology, 12(4), 333-341. https://doi.org/10.1080/17439760.2016.1209542

Park, C. L., \& George, L. S. (2013). Assessing meaning and meaning making in the context of stressful life events: Measurement tools and approaches. The Journal of Positive Psychology, 8, 483-504. https://doi.org/10.1080/17439760.2013.830762

Pulopulos, M. M., \& Kozusznik, M. W. (2018). The moderating role of meaning in life in the relationship between perceived stress and diurnal cortisol. Stress, 21(3), 203210. https://doi.org/10.1080/10253890.2018.1429397

Quiroga, F. L. (2015). O mal-estar na contemporaniedade e suas expressões na docência. Paco Editorial.

Redding, C., \& Henry, G. T. (2019). Leaving school early: An examination of novice teachers' within-and end-of-year turnover. American Educational Research Journal, 56(1), 204-236. http://doi.org/10.3102/0002831218790542

Santos, C. M. D. C., Pimenta, C. A. D. M., \& Nobre, M. R. C. (2007). A estratégia PICO para a construção da pergunta de pesquisa e busca de evidências. Revista Latino-Americana de Enfermagem, 15(3), 508-511. https://doi.org/10.1590/S010411692007000300023

Silva, J. P., Damásio, B. F., \& Melo, S. A. (2009). O sentido de vida e o estresse do professorado: Um estudo correlacional. Cadernos de Psicologia Social do Trabalho, 12(1), 111-122. https://doi.org/10.11606/issn.1981-0490.v12i1p111-122

Souza, G. P. (2017). Inimigos Públicos. Usina Editorial.

Taş, İ., \& İskender, M. (2017). An examination of meaning in life, satisfaction with life, self-concept and locus of control among teachers. Journal of Education and Training Studies, 6(1), 21-31. https://doi.org/10.11114/jets.v6i1.2773

Tomic, W., \& Tomic, E. (2008). Existential fulfillment and burnout among principals and teachers. Journal of Beliefs \& Values, 29(1), 11-27. https://doi.org/10.1080/13617670801928191

Tostes, M. V., Albuquerque, G. S. C. D., Silva, M. J. D. S., \& Petterle, R. R. (2018). Sofrimento mental de professores do ensino público. Saúde em Debate, 42(116), 87-99. http://dx.doi.org/10.1590/0103-1104201811607

Valdanha, E. D., Scorsolini-Comin, F., Peres, R. S., \& Santos, M. A. (2013). Influência familiar na anorexia nervosa: Em busca das melhores evidências científicas. Jornal Brasileiro de Psiquiatria, 62(3), 225-233. https://doi.org/10.1590/s004720852013000300007

Whitaker, R. C., Dearth-Wesley, T., \& Gooze, R. A. (2015). Workplace stress and the quality ofteacher-children relationships in Head Start. Early Childhood Research Quarterly, 30, 57-69. https://doi.org/10.1016/j.ecresq.2014.08.008

Zaidan, J. M., \& Galvão, A. C. (2020). COVID-19 e os abutres do setor educacional: A superexploração da força de trabalho escancarada. In C. B. Augusto, \& R. D. Santos, Pandemias e pandemônio no Brasil (1a. ed., pp. 261-278). Tireant lo Blanch. 
Submetido: 04/03/2021

Reformulado: $15 / 05 / 2021$

Aceite: $20 / 06 / 2021$

\section{Sobre os autores:}

Karine David Andrade Santos é doutoranda em Psicologia pela Universidade Federal de Sergipe.

Joilson Pereira da Silva é doutor em Psicologia pela Universidade Complutense de Madri-Espanha. Professor Associado da Universidade Federal de Sergipe.

Correspondência: psimulti@gmail.com 